\title{
Assessing surgical risk for adults with congenital heart disease: Are pediatric scoring systems appropriate?
}

\author{
Brian Kogon, $\mathrm{MD},{ }^{\mathrm{a}}$ and Matthew Oster, $\mathrm{MD}, \mathrm{MPH}^{\mathrm{b}}$
}

Background: Patients with congenital heart disease are frequently surviving into adulthood, and many of them will require surgery. Currently, there is no validated risk scoring system for adult congenital heart surgery, and predicting outcomes in these patients is challenging. Our objective was to determine if commonly used pediatric congenital heart disease surgery risk scores are also applicable to adults.

\begin{abstract}
Methods: Four hundred fifty-eight adult (age $\geq 18$ years) operations involving cardiac surgery for congenital heart disease between 2000 and 2010 at a single institution were studied retrospectively. The pediatric scores evaluated were the Risk Adjustment for Congenital Heart Surgery (RACHS-1) score, the Aristotle Basic Score, and the Society of Thoracic Surgery-European Association for Cardio-Thoracic Surgery (STAT) Congenital Heart Surgery Mortality score. Receiver operating characteristic (ROC) curves were generated to assess the ability of the scoring systems to predict mortality, major adverse events (stroke, renal failure, prolonged ventilation, prolonged coma, deep sternal infection, reoperation, and operative mortality), and prolonged length of stay ( $>7$ days).
\end{abstract}

Results: Of 458 operations, there were $16(3 \%)$ deaths, $94(21 \%)$ major adverse events, and $90(20 \%)$ prolonged lengths of stay. Four hundred thirty (94\%) of the operations were included in all 3 scoring systems and the ROC analysis. For mortality, areas under the ROC curve were 0.91, 0.91, and 0.65 for the Aristotle, STAT, and RACHS-1 scores, respectively. For major adverse event, areas under the ROC curves were 0.81, 0.76, and 0.61 for the Aristotle, STAT, and RACHS-1 scores, respectively. For prolonged length of stay, areas under the ROC curve were $0.82,0.76$, and 0.61 for the Aristotle, STAT, and RACHS-1 scores, respectively.

Conclusions: Pediatric risk scoring systems such as Aristotle, STAT, and RACHS- 1 offer prognostic value in adults undergoing congenital heart surgery. The scores are predictive of mortality, major adverse events, and prolonged lengths of stay. The STAT and Aristotle systems fared best. (J Thorac Cardiovasc Surg 2014;147:666-71)

Earn CME credits at

http://cme.ctsnetjournals.org

Patients with congenital heart disease are frequently surviving into adulthood. In 2000, the number of adult patients with congenital heart disease in the United States was estimated to be between 650,000 and 1.3 million. ${ }^{1-3}$ More importantly, the prevalence of severe congenital heart disease has increased by $85 \%$ in adults compared with $22 \%$ in children, consistent with the notion that the greatest survival trend has occurred in patients with more

From the Department of Cardiothoracic Surgery, ${ }^{\text {a }}$ Emory University School of Medicine, Atlanta, Ga; and Sibley Heart Center Cardiology, ${ }^{\text {b }}$ Children's Healthcare of Atlanta, Atlanta, Ga.

Disclosures: Authors have nothing to disclose with regard to commercial support.

Read at the 39th Annual Meeting of The Western Thoracic Surgical Association, Coeur d'Alene, Idaho, June 26-29, 2013.

Received for publication June 17, 2013; revisions received Sept 15, 2013; accepted for publication Sept 23, 2013; available ahead of print Nov 18, 2013.

Address for reprints: Brian Kogon, MD, Department of Cardiothoracic Surgery, Emory University Hospital, 1405 Clifton Rd, NE, Atlanta, GA 30322 (E-mail: bkogon@emory.edu).

$0022-5223 / \$ 36.00$

Copyright (c) 2014 by The American Association for Thoracic Surgery

http://dx.doi.org/10.1016/j.jtcvs.2013.09.053 severe disease..$^{1-4}$ As adults, many of these patients with complex conditions will require surgery.

Currently, there is no validated risk scoring system for adult congenital heart surgery, and predicting outcomes in these patients is challenging. In children, the Risk Adjustment for Congenital Heart Surgery (RACHS-1) scoring system, the Aristotle scoring system, and the Society of Thoracic Surgery-European Association for CardioThoracic Surgery (STAT) Congenital Heart Surgery Mortality scoring system have been used to stratify risk and predict/compare outcomes.

The purpose of this study was to determine if commonly used pediatric congenital heart disease surgery risk scores were also applicable to adults. The prognostic value of the pediatric scoring systems with respect to mortality, major adverse events, and length of stay in adult was evaluated in patients with congenital heart disease.

\section{METHODS}

A retrospective cohort study of 458 consecutive operations on adult patients (age $\geq 18$ years) with a previous history of congenital heart disease, who underwent cardiac surgery at a single, large, academic center between January 1, 2000, and December 31, 2010, was performed. For all operations, the surgical procedure was attributed to congenital cardiac pathology. Institutional Review Board approval was obtained for this retrospective study, and individual patient consent was waived. 


\section{Abbreviations and Acronyms \\ EACTS = European Association for \\ Cardiothoracic Surgery \\ RACHS-1 = Risk Adjustment for Congenital Heart Surgery \\ ROC = receiver operating characteristic \\ STAT = Society of Thoracic Surgery-European \\ Association for Cardio-Thoracic \\ Surgery \\ STS $=$ Society of Thoracic Surgeons}

\section{Scoring Systems}

The RACHS-1 scoring system, the Aristotle basic scoring system, and the STAT Congenital Heart Surgery Mortality scoring system were evaluated. Using tables from the landmark articles on the RACHS-1, Aristotle, and STAT scoring systems, we assigned each operation a score within each system. ${ }^{5-7}$ For the RACHS- 1 system, the scale ranged from 1 to $6 .{ }^{5}$ For the Aristotle basic system, the scale ranged from 1.5 to 15 , and a corresponding basic complexity level between 1 and 4 was assigned (level 1 , 1.5-5.6; level 2, 6-7.8; level 3, 8-9.5; level 4, 10-15). ${ }^{6}$ For the STAT score, the scale ranged from 0.1 to 5.0 , and a corresponding mortality category level between 1 and 5 was assigned (level 1, 0.1-0.3; level 2, 0.4-0.7; level 3, 0.8-1.2; level 4, 1.3-2.6; level 5, 2.9-5.0). ${ }^{7}$ In each scoring system, a higher score indicates a higher risk of mortality. For patients undergoing multiple procedures, the procedure with the highest level was scored.

\section{Outcomes}

Operative mortality, presence of a major adverse event, and length of hospital stay greater than 7 days were chosen as the 3 primary outcomes. Operative mortality was defined as a death occurring during the surgical hospital stay or within 30 days of surgery. Because of the infrequency of individual complications, a composite adverse event outcome was used. The major adverse event composite outcome variable was positive if the patient had any of the following major complications: stroke, renal failure, prolonged ventilation, deep sternal infection, reoperation, and operative mortality. All patients were followed until the time of discharge to determine the occurrence of a primary outcome.

The Society of Thoracic Surgeons (STS) database was used to define the list of major adverse events. ${ }^{8}$ Stroke was defined as a neurologic deficit caused by a disturbance in cerebral perfusion that did not resolve within 24 hours. Renal failure was defined by a serum creatinine level greater than 2.0 and twice the preoperative level, or by a new requirement for dialysis. Prolonged ventilation was defined as any requirement for mechanical ventilation more than 24 hours postoperatively. Deep sternal infection was defined as a sternal infection within 30 days of surgery, requiring all of the following conditions: (1) wound or mediastinal exploration with excision of tissue, (2) positive wound culture, and (3) treatment with antibiotics. Reoperation was defined as surgery performed for any reason within 30 days of surgery. Operative mortality included (1) all deaths occurring during the hospital stay when the operation was performed and (2) those deaths occurring after discharge from the hospital, but within 30 days of the procedure.

\section{Analysis/Statistics}

The occurrences of mortality, major adverse events, and prolonged lengths of stay were summarized for the entire cohort. For those patient operations with a score from each scoring system, logistic regression was used to generate receiver operating characteristic (ROC) curves to assess the ability of each scoring system to predict each of the 3 primary
TABLE 1. Frequency of operations performed (in descending order)

\begin{tabular}{lr}
\hline \multicolumn{1}{c}{ Procedure name } & n \\
\hline Pulmonary valve repair & 142 \\
Tricuspid valve repair & 29 \\
Conduit reoperation & 28 \\
Atrial septal defect repair, patch & 25 \\
Aortic root replacement, mechanical or bioprosthetic & 24 \\
Valvuloplasty, tricuspid & 19 \\
Atrial septal defect repair, patch + partial anomalous & 16 \\
pulmonary venous connection repair &
\end{tabular}

pulmonary venous connection repair

Fontan revision or conversion (redo Fontan procedure) 16

Mitral valve repair $\quad 15$

Transplantation, heart $\quad 14$

Valvuloplasty, mitral 14

Aortic valve replacement, mechanical or bioprosthetic 11

$\begin{array}{ll}\text { Pulmonary artery, reconstruction (plasty), branch, central } & 10\end{array}$

(within the hilar bifurcation)

Aortic valve closure (aortic valve septal defect) repair, $\quad 8$ partial (incomplete)

Double chamber right ventricle repair

Ventricular septal defect repair, patch

Coronary artery bypass

Ross procedure

Coarctation repair, end-end

Valvuloplasty, aortic

Aortic stenosis, supravalvular, repair

Cardiac tumor resection

Anomalous origin of coronary artery repair

Aortic aneurysm repair

Arrhythmia surgery, atrial, surgical ablation

Coarctation repair, interposition graft

Partial anomalous pulmonary venous connection, scimitar, repair

Anomalous systemic venous connection repair 2

Aortic arch repair

Aortic stenosis, subvalvular, repair

Bidirectional cavopulmonary anastomosis

(bidirectional Glenn procedure)

Ventricular assist device

8

Aortic arch repair + ventricular septal defect repair

Aortic dissection repair

Aortic root replacement, homograft

Aortic root replacement, valve sparing

Atrial septal defect partial closure

Konno procedure

Left atrial appendage excision

Patent ductal arteriosus closure, surgical

Pericardiectomy

Right ventricular outflow tract procedure

Septal myectomy

Shunt, systemic to pulmonary artery, modified Blalock-Taussig shunt

Sinus of Valsalva, aneurysm repair

Transplantation, lung(s)

Total

outcomes. For the Aristotle and STAT systems, the raw scores were used. Higher area under the ROC curve indicated better discriminatory 


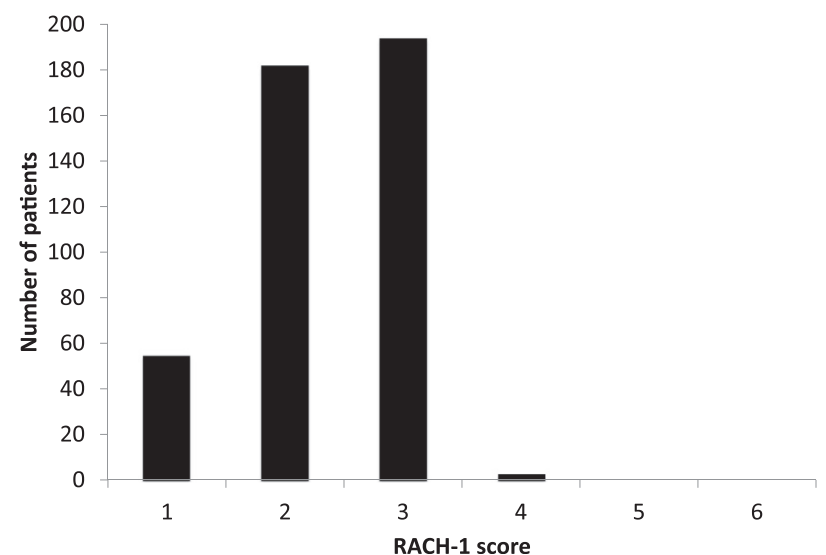

FIGURE 1. RACHS-1 scoring system distribution. RACHS, Risk Adjustment for Congenital Heart Surgery.

performance of the scoring system. ROC curves were compared using a test of joint equality of ROC curve areas. ${ }^{9}$ All statistics were performed using SAS 9.3 (SAS Institute, Inc, Cary, NC).

\section{RESULTS}

Of 458 patient operations, there were $16(3 \%)$ deaths, 94 $(21 \%)$ major adverse events, and $90(20 \%)$ prolonged lengths of stay. A breakdown of the major adverse events showed the following: stroke $(6,1.3 \%)$, renal failure $(36,7.9 \%)$, prolonged ventilation $(63,13.8 \%)$, deep sternal infection $(0)$, reoperation $(58,12.7 \%)$, and operative mortality $(16,3.5 \%)$.

A complete list of the operations and associated frequencies is shown in Table 1. Four hundred thirty $(94 \%)$ operations were included in all 3 scoring systems and the ROC analysis. The scoring distributions among the RACHS-1, Aristotle, and STAT systems are shown in Figures 1 to 3. Some patients could not be scored within the RACHS-1 (24, 5.2\%), Aristotle $(2,0.4 \%)$, and STAT $(5,1.1 \%)$ scoring systems.

\section{Mortality}

For mortality, areas under the ROC curve were 0.91, 0.91, and 0.65 for the Aristotle, STAT, and RACHS-1 scores, respectively (Figure 4, A). Compared with the Aristotle score, there was no difference in the STAT score $(P=.97)$, whereas the RACHS-1 score performed significantly worse $(P<.0001)$.

\section{Major Adverse Events}

For major adverse events, the areas under the ROC curve were 0.81, 0.76, and 0.61 for the Aristotle, STAT, and RACHS-1 scores, respectively (Figure 4, B). The Aristotle score performed significantly better than either the STAT score $(P=.03)$ or the RACHS-1 score $(P<.0001)$.

\section{Length of Stay}

For prolonged length of stay, the areas under the ROC curve were $0.82,0.76$, and 0.61 for the Aristotle, STAT, and RACHS-1 scores, respectively (Figure 4,C). The Aristotle score performed significantly better than either the STAT score $(P=.01)$ or the RACHS-1 score $(P<.0001)$.

\section{Comparison}

For each scoring system, a comparison was also performed between mortality rates in this adult cohort and a similar pediatric cohort at our institution (Table 2). Although the trend is increasing mortality with increasing scores in both populations, the absolute mortality is generally higher in the adult cohort than the pediatric cohort throughout.

\section{DISCUSSION}

In this retrospective cohort study, the first of its kind to evaluate potential scoring systems in congenital heart surgery in an exclusively adult population, we found that scoring systems used in the pediatric population may have prognostic value in adults. Specifically, the Aristotle score fared particularly well in predicting outcomes pertaining to mortality, major adverse events, and length of stay in adults undergoing congenital heart surgery. The STAT score similarly performed well, although not as well as the

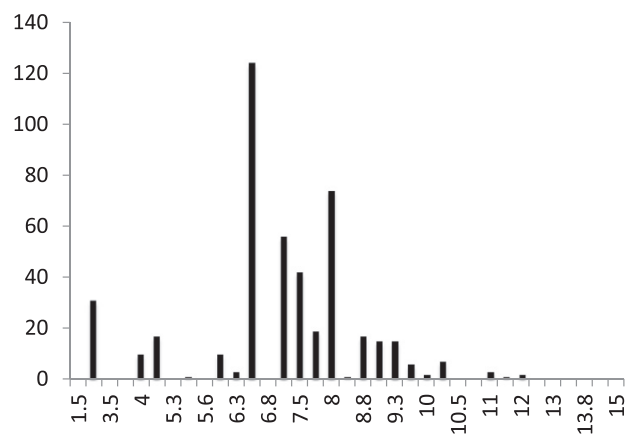

Aristotle basic complexity score

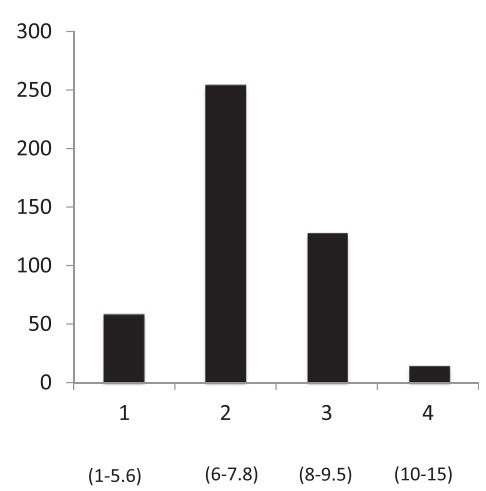

Aristotle basic complexity category

FIGURE 2. Aristotle scoring system distribution. 


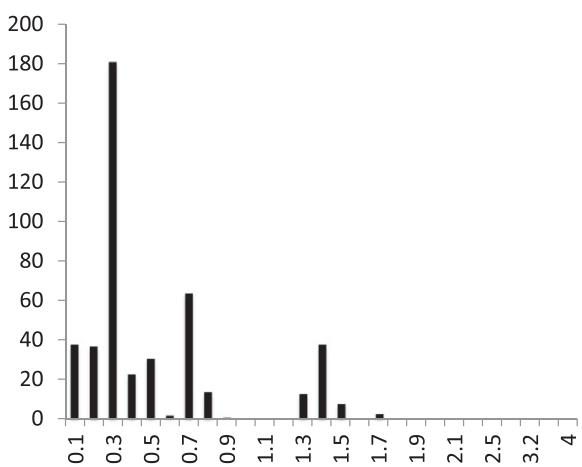

STAT mortality score

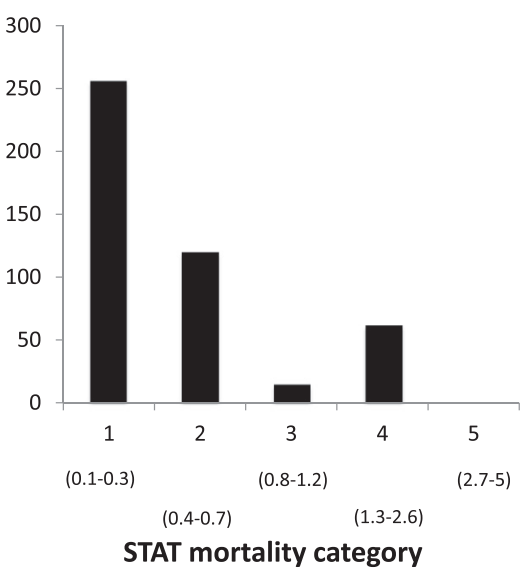

FIGURE 3. STAT scoring system distribution. STAT, Society of Thoracic Surgery-European Association for Cardio-Thoracic Surgery.

Aristotle score in the domains of adverse events and length of stay. The RACHS-1 score, although of some prognostic value, did not function as well as the other scoring systems.

\section{Current Tools in Pediatric Congenital Heart Surgery}

Over the last 2 decades, pediatric cardiac surgeons have recognized the need for reliable outcome reporting, as shown by the creation of several congenital heart surgery databases and several risk stratification scoring systems. Nonetheless, it remains challenging when the individual diagnoses and surgical procedures are numerous and the number of individual patients is small. Despite this, several tools have been developed to measure outcomes, compare performance, and provide a platform for quality improvement in pediatric congenital heart surgery.

RACHS-1 was first described in 2001. A panel of pediatric cardiologists and pediatric cardiac surgeons used clinical judgment to place surgical procedures into 6 risk categories. The aim was to develop a consensus-based method of risk adjustment for in-hospital mortality among children younger than 18 years after surgery for congenital heart disease. In the initial report, mortality rates were $0.4 \%$ in category $1,3.8 \%$ in category $2,8.5 \%$ in category 3 , $19.4 \%$ in category 4 , and $47.7 \%$ in category 6 . There were too few cases in category 5 to estimate mortality rates. $^{5}$

The Aristotle scoring system was described in 2004. A panel of expert surgeons representing the European Association for Cardiothoracic Surgery (EACTS), STS, European Congenital Heart Surgeons Association, and Congenital Heart Surgeons Society, assigned each operation 2 scores. The first score was the Basic Score, which adjusted only for the complexity of the procedure. The second score was the Comprehensive Score, which took into account specific procedure-dependent and
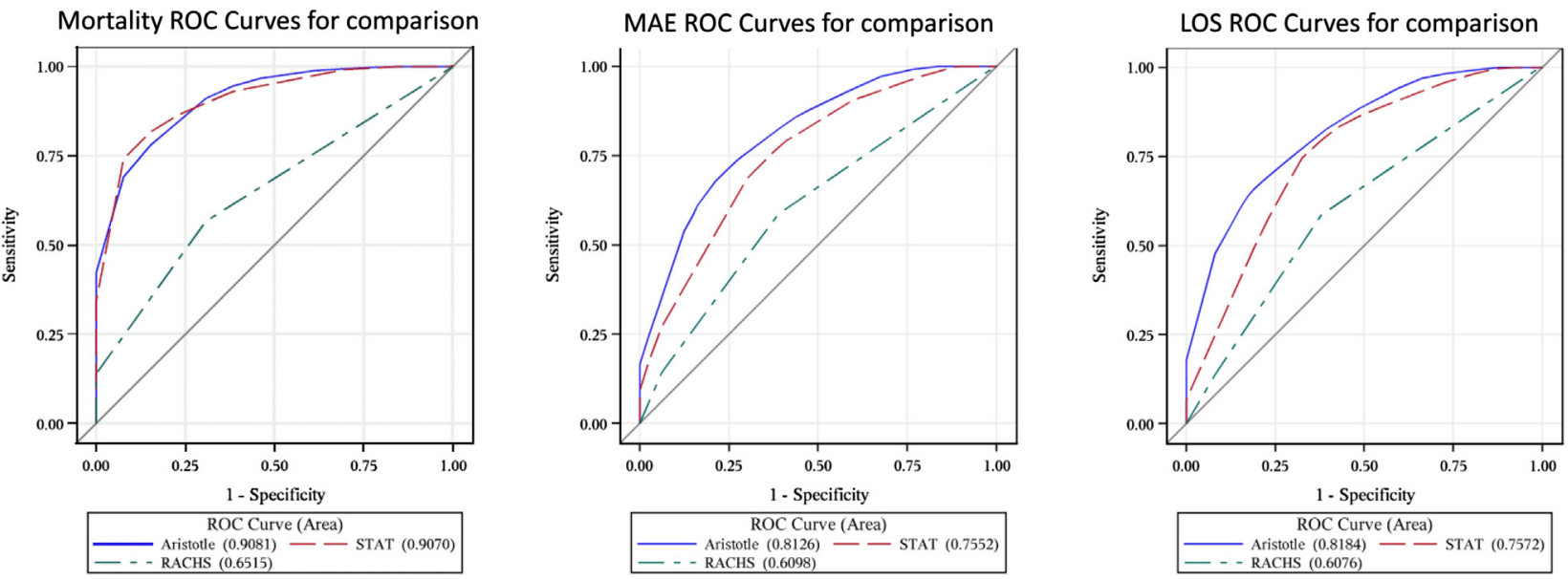

FIGURE 4. ROC curves. A, Mortality ROC curves for comparison. B, Major adverse event ROC curves for comparison. C, Length of stay ( $L O S)$ ROC curves for comparison. ROC, Receiver operator characteristic; MAE, major adverse event; STAT, Society of Thoracic Surgery-European Association for Cardio-Thoracic Surgery; RACHS, Risk Adjustment for Congenital Heart Surgery. 
TABLE 2. Comparison of adult and pediatric mortality

\begin{tabular}{ccc}
\hline $\begin{array}{c}\text { Scoring } \\
\text { system }\end{array}$ & $\begin{array}{c}\text { Adult cohort } \\
\text { mortality (\%) }\end{array}$ & $\begin{array}{c}\text { Pediatric cohort } \\
\text { mortality (\%) }\end{array}$ \\
\hline RACHS-1 & 0 & 0 \\
1 & 2.2 & 0 \\
2 & 4.6 & 1.4 \\
3 & 0 & 3.8 \\
4 & na & 13.6 \\
$5-6$ & & \\
STAT & 0.3 & 0 \\
1 & 5 & 0.6 \\
2 & 6.6 & 1.7 \\
3 & 9.7 & 4.2 \\
4 & na & 12.5 \\
5 & & \\
Aristotle & 0 & 0 \\
1 & 3.3 & 1.4 \\
2 & 6.2 & 2 \\
3 & 0 & 4.2 \\
4
\end{tabular}

na, Not applicable; STAT, Society of Thoracic Surgery-European Association for Cardio-Thoracic Surgery; RACHS, Risk Adjustment for Congenital Heart Surgery.

procedure-independent patient characteristics. The Basic Score ranged from 1.5 to 15 and 4 levels of difficulty were defined. ${ }^{6}$

In 2010, a new scoring system (STS-EACTS, or STAT) was developed to create an objective index, as opposed to the RACHS-1 and Aristotle systems, which were predominantly subjectively derived. Mortality risk was estimated using data from operations entered into the EACTS Congenital Heart Surgery Database and the STS Congenital Heart Surgery Database. The objectives of this new system were to (1) estimate procedure-specific relative risks of in-hospital mortality, (2) convert these procedurespecific mortality estimates into a scale ranging from 0.1 to 5.0 (STAT mortality score), and (3) group procedures with similar estimated mortality into a small number of relatively homogeneous categories (STAT mortality category).

Although these scoring systems were designed primarily to predict mortality, they also have prognostic value for other outcomes. Studies have shown correlation with higher-risk procedures and postoperative infection, prolonged mechanical ventilation, feeding difficulty, acute renal failure requiring dialysis, and readmissions after pediatric congenital heart surgery. ${ }^{10-14}$

\section{Current Tools in Adult Congenital Heart Surgery}

The STS risk calculator and the RACHS-1, Aristotle, and STAT scoring systems have been instrumental in outcome reporting, performance comparison, and quality improvement initiatives in adult-acquired heart surgery and pediatric congenital heart surgery, respectively. Unfortunately, the available tools for evaluating risk and predicting outcome in adult congenital heart surgery are limited. The current version of the STS risk calculator does not allow for entry of most congenital operations (only coronary bypass operations, aortic or mitral valve operations, or some combination thereof), and it is unclear whether the established pediatric congenital scoring systems apply to adult patients with congenital heart disease. As a result, predicting outcomes in these patients is challenging.

The distribution of operations is not the same in the 2 patient populations. Using the currently available risk factor scoring systems, the distributions in our pediatric congenital cardiac operations and our adult congenital cardiac operations are different; the distribution is skewed toward the lower-risk procedures in adults (Figure 5). In our adult congenital population, few patients were in the higher-risk category (ie, Norwood operation, interrupted aortic arch, truncus arteriosus), whereas many patients were in the lower- and middle-risk categories (ie, pulmonary valve replacement, aortic valve replacement). In addition to the distribution of operations, the patientspecific characteristics and comorbidities that may influence outcomes are also different between the adult and pediatric patient populations. For example, based on the Aristotle Comprehensive Scoring system, factors that may influence outcome in the pediatric population include low birth weight, prematurity, genetic and chromosomal
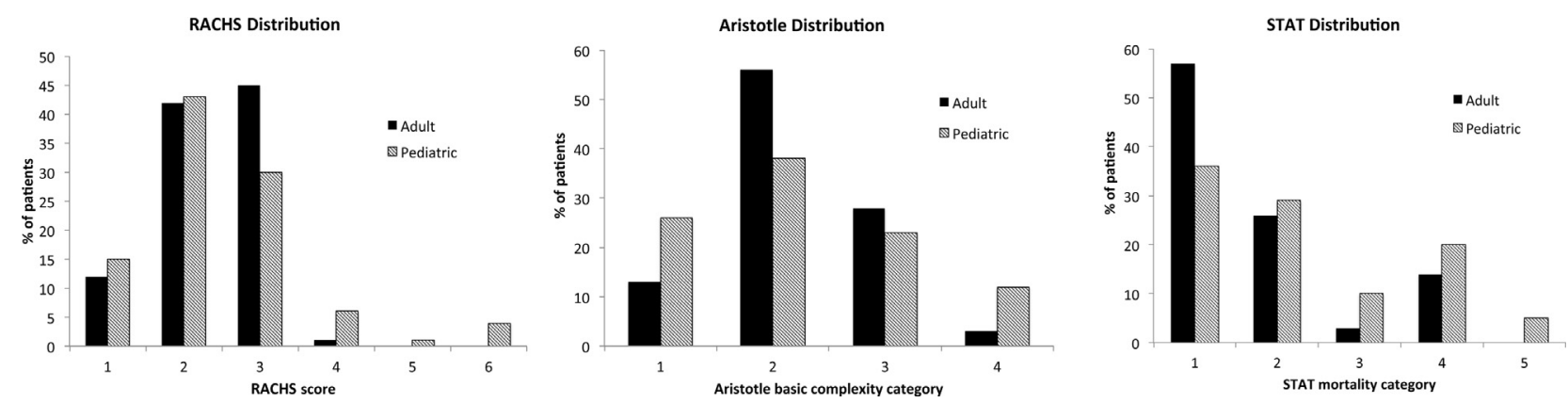

FIGURE 5. Adult and pediatric case distribution within each scoring system. RACH, Risk Adjustment for Congenital Heart Surgery; STAT, Society of Thoracic Surgery-European Association for Cardio-Thoracic Surgery. 
issues, and noncardiac congenital anomalies. ${ }^{6}$ Based on the STS risk calculator for acquired adult cardiac surgery, factors that may influence outcome in the adult population include diabetes mellitus, hypertension, peripheral vascular disease, and New York Heart Association class. ${ }^{8}$

With these differences in mind, our objective was to determine if commonly used pediatric congenital heart disease surgery risk scoring systems were also applicable to adult patients with congenital heart disease. From our study, it seems that despite the differences in the pediatric and adult populations with congenital heart disease, pediatric risk scoring systems can predict mortality in adult patients undergoing congenital surgery. Just as in the pediatric population, they also seem to have prognostic value with respect to other outcomes, such as major adverse events and prolonged length of stay. As shown, the Aristotle and STAT scoring systems are better than the RACHS-1 system in predicting these adverse outcomes. The Aristotle and STAT scoring systems likely fared better because of the wider distribution of adult operations for congenital heart conditions across the scoring spectrum compared with the RACHS-1 system.

\section{Limitations}

This study is limited by its observational nature and the inherent limitations of a retrospective database study. Also, these scoring systems, particularly the Aristotle system, take into account additional procedure- and patient-specific factors that were not included in the analysis.

\section{CONCLUSIONS}

Although there is currently no validated scoring system for congenital heart surgery in adults, the risk stratification systems for similar surgeries in children do have some prognostic value; the Aristotle and STAT scoring systems fared the best. Future efforts are warranted to further optimize these or similar scoring systems to obtain even better prognostic value in the adult population with congenital heart disease.

\section{References}

1. Hoffman JI, Kaplan S. The incidence of congenital heart disease. J Am Coll Cardiol. 2002;39:1890-900.

2. Hoffman JI, Kaplan S, Liberthson RR. Prevalence of congenital heart disease. Am Heart J. 2004;147:425-39.

3. Marelli AJ, Gurvitz M. From numbers to guidelines. Prog Cardiovasc Dis. 2011; 53:239-46.

4. Warnes CA, Liberthson R, Danielson GK, Dore A, Harris L, Hoffman JI, et al. Task force 1: the changing profile of congenital heart disease in adult life J Am Coll Cardiol. 2001;37:1170-5.

5. Jenkins K, Gauvreau K, Newberger J, Spray T, Moller J, Iezzoni L. Consensus-based method for risk adjustment for surgery for congenital heart disease. J Thorac Cardiovasc Surg. 2002;123:110-8.

6. Lacour-Gayet F, Clarke D, Jacobs J, Comas J, Daebritz S, Daenen W, et al., and the Aristotle Committee. The Aristotle score: a complexity-adjusted method to evaluate surgical results. Eur J Cardiothorac Surg. 2004;25:911-24.

7. O'Brien S, Clarke D, Jacobs J, Jacobs M, Lacour-Gayet F, Pizarro C, et al. An empirically based tool for analyzing mortality associated with congenital heart surgery. J Thorac Cardiovasc Surg. 2009;138:1139-53.

8. Society of Thoracic Surgeons. Online STS risk calculator. Available at: http:// riskcalc.sts.org/STSWebRiskCalc273/support_definitions.html. Accessed May $1,2013$.

9. DeLong ER, Delong DM, Clarke-Pearson DL. Comparing the areas under two or more correlated receiver operating characteristic curves: a non-parametric approach. Biometrics. 1988;44:837-45.

10. Kansy A, Jacobs JP, Pastuszko A, Mirkowicz-Malek M, Manowska M, Jezierska E, et al. Major infection after pediatric cardiac surgery: external validation of risk estimation model. Ann Thorac Surg. 2012;94:2091-5.

11. Shi S, Zhao Z, Shu Q, Tan L, Lin R, Shi Z, et al. Perioperative risk factors for prolonged mechanical ventilation following cardiac surgery in neonates and young infants. Chest. 2008;134:768-74.

12. Kogon B, Ramaswamy V, Todd K, Plattner C, Kirshbom PM, Kanter KR, et al. Feeding difficulty in newborns following congenital heart surgery. Congenit Heart Dis. 2007;2:332-7.

13. Pederson KR, Povlsen JV, Christensen S, Pederson J, Hiortholm K, Larsen SH, et al Risk factors for acute renal failure requiring dialysis after surgery for congenital heart disease in children. Acta Anaesthesiol Scand. 2007;51:1344-9.

14. Kogon B, Jain A, Oster M, Woodall K, Kanter K, Kirshbom P. Risk factors associated with readmission following pediatric cardiothoracic surgery. Ann Thorac Surg. 2012;94:865-73. 